\title{
MULTIFRACTAL APPROACH TO THE ANALYSIS OF CRIME DYNAMICS: RESULTS FOR BURGLARY IN SAN FRANCISCO
}

\author{
MIGUEL MELGAREJO ${ }^{*, \dagger}$ and NELSON OBREGON ${ }^{\dagger, \ddagger}$ \\ *Universidad Distrital Francisco José de Caldas \\ Bogotá DC, Colombia \\ ${ }^{\dagger}$ Pontificia Universidad Javeriana, Bogotá DC, Colombia \\ ${ }^{\ddagger}$ Universidad Nacional de Colombia, Bogotá DC, Colombia \\ Received November 16, 2016 \\ Accepted May 4, 2017 \\ Published August 18, 2017
}

\begin{abstract}
This paper provides evidence of fractal, multifractal and chaotic behaviors in urban crime by computing key statistical attributes over a long data register of criminal activity. Fractal and multifractal analyses based on power spectrum, Hurst exponent computation, hierarchical power law detection and multifractal spectrum are considered ways to characterize and quantify the footprint of complexity of criminal activity. Moreover, observed chaos analysis is considered a second step to pinpoint the nature of the underlying crime dynamics. This approach is carried out on a long database of burglary activity reported by 10 police districts of San Francisco city. In general, interarrival time processes of criminal activity in San Francisco exhibit fractal and multifractal patterns. The behavior of some of these processes is close to $1 / f$ noise. Therefore, a characterization as deterministic, high-dimensional, chaotic phenomena is viable. Thus, the nature of crime dynamics can be studied from geometric and chaotic perspectives. Our findings support that crime dynamics may be understood from complex systems theories like self-organized criticality or highly optimized tolerance.
\end{abstract}

Keywords: Crime Patterns; Crime Dynamics; Complexity; Multifractal Analysis; Chaos; $1 / f$ Noise.

\footnotetext{
This is an Open Access article published by World Scientific Publishing Company. It is distributed under the terms of the Creative Commons Attribution 4.0 (CC-BY) License. Further distribution of this work is permitted, provided the original work is properly cited.
} 


\section{INTRODUCTION}

Through observation and analysis, crime is recognized as a complex phenomenon! It has been noticed in the macroscopic scale that crime is ubiquitous to urban systems and is not uniformly distributed. It has been also noted that small changes introduced by criminal activity at the microscopic level lead to noticeable changes in the environment of cities. Crime pattern theory ${ }^{2]}$ considers crime to be a complex phenomenon that forms patterns in space and time.

One of the main formulations of pattern theory is that crime does not occur or distribute randomly. This perspective marks an epistemological background that invites formulation of concepts and rules that account for the non-randomness of crime dynamics seen in reality ${ }^{3}$ A pattern can be understood as the possible interconnection between objects, rules or processes, which can be physical or conceptual. Patterns can be established from evidence or can be given from theoretical formulations. In some cases patterns may be obvious, but in other cases it is necessary to delve deeper into the environment as a whole to detect them.

Routine activity theory ${ }^{4}$ primarily focuses on the interaction between the victim and offender in a certain time and place. This theory suggests that the interaction is made possible due to the absence of factor inhibitors as guardians or authorities. $\frac{5}{5}$ These factors can be understood as restrictions on the criminal opportunity. The emphasis of the theory rests on victims bearing in mind that criminal opportunities would depend on the availability of targets. The execution of a criminal offense depends on the benefits that the offender can receive, if these outweigh the risks of getting caught. Thus, crime patterning is influenced by the social relations of offenders and their interaction with the environment where they develop their daily activities. This aspect indicates that crime dynamics is connected with the complex dimension of social systems and their actors. 6 ]

Probability and statistics, as well as stochastic process theory, are found in the core of the quantitative analysis of crime dynamics. ${ }^{[7}[9]$ Other epistemological approaches, such as fuzzy set theory 10 11 and complex network theory ${ }^{12}$ have been applied to cope with the inherent uncertainty and complexity of this phenomenon. Chaos theory has been considered an interesting apparatus to clarify several problems in criminology 13 Several studies analyze crime dynamics by using nonlinear approaches close to this theoretic perspective ${ }^{14 \mid 15}$ However, quantitative analysis of criminal data based on chaos theory and tools is still an open issue. In addition, determining how crime behaves in space ${ }^{16}$ or space-time ${ }^{17}$ following a dynamical perspective is an important topic in crime research.

When dealing with complexity, some intriguing words come to mind such as uncertainty or irregularity. These words flourish when inquiring for a certain class of phenomena in which there is a loss of information or an abundance of variables and relations between these. Although nowadays there is not a single definition for the term complexity, nor could there be considered a simple metaphor or an elegant way to talk about certain phenomena. It can be observed in this category that it is rooted in nature, becoming latent where multiple entities interact with each other as processes ${ }^{18}$ Complexity would then refer to a type of dynamic characterized by multiplicity. 19

The following properties depict the footprint of complexity in a system 20121 .

- Nonlinearity: The principles of uniformity and superposition do not apply in complex systems. These systems are characterized by sensitivity to initial conditions and behaviors that, without being periodic, exhibit some degree of recurrence. In addition, in systems composed of multiple units, it is typical to deal with a particular form of chaos that comes from the variability induced by the interactions between these units.

- Emergency: In a system that is perceived as complex, some global properties manifest depending on the system as a whole and cannot be explained or reduced in terms of the individual parts which constitute it. The term unexpected behavior may be used in this context as synonymous with emergent property.

- Self-organization: The non-reductionist nature of a complex system facilitates the emergence and sustenance of hierarchical structures that would control the disorder of small scales. In this way, the system itself is able to organize in states of order and long-range coherence.

- Unpredictability: The perception of order and disorder, the nesting of different scales of analysis and the elements of surprise make the observer seriously wonder about the possibility of predicting the dynamics of a complex system. The unpredictability is related to the system itself (i.e. chaos 
or edge of chaos) but at the same time to the inability of those observing it to determine where it will evolve. 19

The approximation to complex social systems should imply an epistemological development that captures the evolution of the system dynamics. 22 The multiple transformations that may arise from adaptation in a social system make its understanding only in terms of its agents too narrow. Therefore, the category of complex systems demands an analytical approach not only from its parts but also from the statistical attributes that are involved in its dynamics. The question about the relationship between some of these characteristics, such as power laws, $1 / f$ noise, fractal hierarchies and chaotic behaviors, demands to be asked about the theoretical and empirical aspects of phenomena where these properties may appear. ${ }^{23}$ This background will guide the discussion about the possible relationships behind these attributes and crime in the context of complex systems analysis. This is supported by previous evidence showing the emergence of power laws in crime ${ }^{6[24}$

A brief description about the mathematical principle that explains the emergence of power laws will be presented. Then, two possible theories that explain power laws in several phenomena will be introduced $\stackrel{25}{25}$ One of them, the self-organized criticality (SOC), offers an interesting link between power laws and $1 / f$ noise ${ }^{26]}$ Some properties related to this class of noise will be discussed in the light of stochastic processes theories, which allow connecting the $1 / f$ noise with the concept of fractal process and its characterization by means of the Hurst exponent. 27 Zipf's laws and $1 / f$ spectra will be summarized, highlighting that these properties can be organized as a multifractal hierarchy. This concept points out that hierarchical power laws are a footprint of complexity. The evidence of fractal and multifractal properties prompts the investigation of other statistical attributes related to chaos and information theory ${ }^{28 \mid 29}$ marking an epistemological context that goes beyond the probabilistic approach to treat the inherent uncertainty.

The basis for a complexity approach to the analysis of criminal data is that crime dynamics is characterized by (1) patterning in space and time and (2) emerging from complex social systems. The key idea behind this framework is the fact that fractal and multifractal analyses, as well as chaos-based tools, do not require assumptions of ergodicity or statistical independence. In addition, this framework suggests a way to visualize informational patterns in crime dynamics. This last point will be illustrated by showing that a burglary record of 13 years in San Francisco City exhibits multifractality, chaos and informational patterns.

\section{METHODS}

\subsection{Power Laws}

The Zipf's law belongs to the family of distributions that behave according to a power law expressed by

$$
p(x)=C x^{-\propto},
$$

where $\propto$ is the power law exponent and $C$ is a scaling constant that guarantees the requirement of the sum of $p(x)$ to be 1.0. Of these parameters, $\propto$ is the most important when characterizing the distribution.

Distributions that follow power laws such as Eq. (1) are characterized by exhibiting the scale-free property. This means that the shape of the distribution remains the same, except from a multiplicative constant, when a rescaling of the independent variable is introduced 25 .

$$
p(b x)=g(b) p(x) .
$$

It follows that

$$
\frac{d p}{d x}=\frac{p^{\prime}(1)}{p(1)} p(x),
$$

where the solution to this differential equation behaves as

$$
\operatorname{Ln}(p(x))=\frac{p(1)}{p^{\prime}(1)} \operatorname{Ln}(x)+k .
$$

It can be verified that the solution to (4) is

$$
p(x)=p(1) x^{-\alpha},
$$

where $\alpha=p(1) / p^{\prime}(1)$ corresponds to the behavior expressed by Eq. (1). This shows that a power law distribution is accomplished naturally with the scale invariance property.

Several theories have been proposed from the physics domain to explain why diverse phenomena produce power laws. One of these theories, the highly optimized tolerance (HOT) explanation, suggests that extraordinary events appear because of the interaction of designs that favor small and frequent losses whose statistics are predictable from big losses that arise in rare perturbations. ${ }^{[30}$ Designs can be obtained from processes like natural selection or intentional human activity. These designs 
result in systems that are tolerant to significant fluctuations of the environment but are seriously sensible to microscopic perturbations. For example, while complex organisms tolerate big fluctuations of environment conditions, they succumb easily if their regulatory systems are affected by microorganisms, toxins or small wounds.

The SOC theory proposes that the length of the correlation between microscopic interactions in a system becomes larger than the range in which they are produced ${ }^{26}$ Thus, the system behavior turns independent from the microscopic details. The systems in which it occurs are characterized by sustaining a constant flux of matter and energy from and to their surrounding environment. Neuronal avalanches, earthquakes and forest fires are the kind of phenomena that might be explained by this theory.

\subsection{Long-Range Dependence and $1 / f$ Noise}

The stochastic processes characterized as $1 / f$ noise exhibit a power spectral density given by

$$
S(f)=S_{o} f^{-\beta},
$$

where $f$ is the frequency, $\beta$ is the spectral exponent and $S_{0}$ is a scaling constant of the process.

The spectral shape given by Eq. (6) arises from the temporal features of the process. Hence, this process $X(t)$ exhibits long-range dependence whose autocorrelation function decays asymptotically as

$$
R_{X}(k)=c k^{-b} \text { with } k \rightarrow \infty,
$$

where $0<b<1$ and $c>0$ so that $R_{X}(k)$ is not summable:

$$
\sum_{k=-\infty}^{\infty} R_{X}(k)=\infty .
$$

According to the Wiener-Khinchin theorem, $\frac{31}{21}$ the relation between the autocorrelation functions of the process and its power spectral density can be expressed as

$$
T(\omega)=\frac{1}{2 \pi} \sum_{k=-\infty}^{\infty} R_{X}(k) e^{-j k \omega} .
$$

Therefore, in practical terms, the spectral power density of a process can be estimated from its autocorrelation function. Thus, the processes with longrange dependence autocorrelation are characterized by exhibiting a power spectral density as in Eq. (6).
The characterization of a process as $1 / f$ noise can be achieved by estimating its Hurst exponent $H$ [27 This parameter is computed from the proportionality exhibited by the changes in the estimation of the process variance with respect to the scale. This proportionality is possible due to the self-similarity of the process at different scales in the statistical sense (i.e. fractal process). This particularity is directly connected to the autocorrelation structure with long-range dependence. For this kind of process, the Hurst parameter is accomplished with $0.5<H<1.0$.

Thus, characterizing a process as fractal can be achieved by detecting one of the following properties:

- Hurst exponent $H$, with $0.5<H<1.0$.

- Long-range dependence autocorrelation structure according to Eq. (7).

- Power spectral density of $1 / f$ noise, according to Eq. (6).

From the perspective of the SOC theory, $1 / f$ noise and fractal structures are related to systems that exhibit several degrees of freedom extended over the space, which evolve naturally toward selforganized structures that are barely stable ${ }^{26}$ In this sense, the system becomes stable when the noise induced by microscopic fluctuations cannot propagate to infinite distances. Thus, the system selforganizes to a critical point where its correlation structure exhibits a power law. A typical example here is the sand pile. If the slope of the pile is too large, it is far from the equilibrium, so it collapses until the average slope reaches a point barely stable with respect to small perturbations. Therefore, $1 / f$ noise can be explained as a dynamical response of the sand pile to microscopic random perturbations.

\subsection{Multifractal Analysis}

According to the SOC theory, there is a deep connection between $1 / f$ noise and the development of fractal patterns in complex systems. These patterns tend to form hierarchies characterized by cascadetype structures and scale invariance. The latter is a common feature of the properties commented on in previous sections. The basic fractal structure recognized in the Cantor set is regarded as a sole mathematic construction; however, the multifractal structure of the generalized Cantor set can be applied to model-free scale phenomena in complex systems. 
Multifractals appear as the result of an interweaving of fractal subsets that exhibit different scaling exponents. In regard to this structure, the zipf's laws or the $1 / f$ spectra can be organized in hierarchies as well. From a self-similar hierarchy, it can be shown that zipf's power laws and $1 / f$ processes can be studied with a common mathematic structure. Thus, according to Ref. 23, it is necessary to show that these laws or spectra organized as harmonic cascade satisfy

$$
S_{m}=N_{m} P_{m}=\text { const., }
$$

where $m$ is the level of the cascade, $N_{m}$ is the number of elements, $P_{m}$ is the average size of the elements and $S_{m}$ is the sum of all elements of a given level. In the case of a hierarchy that follows a harmonic sequence, it is demonstrated that when $\rightarrow \infty$ :

$$
S_{m}=N_{m} P_{m}=\operatorname{Ln}(2) .
$$

This result suggests the equivalence between the harmonic sequence, zipf's laws and $1 / f$ spectra appears when $m$ is large enough. Therefore, when transforming a rank-size distribution or frequency spectrum into a self-similar hierarchy, some differences will be noticed in the first classes of the hierarchy.

It can be observed that there is a hierarchical power law behind zipf's laws, $1 / f$ spectra and selfsimilar hierarchies in the form

$$
y_{m}=\eta x_{m}^{-D},
$$

where $y$ is a sample of the elements in level $m, x$ is the size of the elements in level $m$ and $D$ is the fractal dimension of the hierarchy.

Multifractal analysis can be complemented by computing the multifractal spectrum. ${ }^{32}$ A practical computation can be found in Ref. 33. The multifractal spectrum $f(\alpha)$ is related to the generalized dimension $D(q)$ :

$$
D(q)=\frac{1}{q-1}[q \alpha(q)-f(\alpha(q))],
$$

where $q \in R$ is called the moment of order $q$ and $\alpha(q)$ is the local Lipschitz-Hölder exponent given by

$$
\alpha(q)=\frac{d \tau_{q}}{d q} .
$$

Therefore, the $f(\alpha)$ function can be obtained by evaluating $\tau(q)$ (i.e. the correlation exponent) as

$$
\tau(q)=\operatorname{Lim}_{\varepsilon \rightarrow 0} \frac{\log Z_{q}(\varepsilon)}{\log \varepsilon} .
$$

The observable has a multifractal distribution $Z_{q}(\varepsilon)$ which scales with $\varepsilon$ as $Z_{q}(\varepsilon) \sim \varepsilon^{\tau_{q}}$. A multifractal spectrum is coherent as long as it satisfies the following conditions:

- $D(0) \geq D(1) \geq D(2)$,

- $f(\alpha)$ must be concave,

- $f(\alpha)$ must be tangent to the identity $f(\alpha)=\alpha$,

- $f(\alpha)=\alpha=D(1)$,

where $D(0), D(1)$ and $D(2)$ correspond to the capacity dimension, the information dimension and the correlation dimension, respectively.

\subsection{Observed Chaotic Data}

Chaos as an attribute of an observable signal or process $x(t)$ is a property that manifests itself as complex dynamical traces in time, broadband power density spectra, non-periodic motion and exponential sensitivity to reduced perturbations in the orbit of the phase plane.19 Thus chaos is irregular in time and slightly predictable, but it has structure in phase space. ${ }^{34}$ Most of the work in chaos theory focuses on the analysis of dynamical systems whose state variables and governing equations are known. However, some work is devoted to inferring the chaotic nature of a system by only using data drawn from it. This becomes useful when there is no a priori knowledge about the structure of the system. 35

Chaos detection over an observable (i.e. discrete time series) is plausible thanks to Takens embedding theorem, $\frac{36}{}$ which allows for reconstructing the geometric structure of the multivariate dynamics where the observable exists. A dynamical system can be expressed as

$$
\mathbf{x}(n) \rightarrow F(\mathbf{x}(n))=\mathbf{x}(n+1),
$$

where $\mathbf{x}(t)$ is a multidimensional phase space. The theorem states that if a single scalar quantity $h(\circ)$ of some vector function of the dynamical variables $g(\mathbf{x}(n))$ is available, then the geometry of the dynamics can be unfolded from the scalar measurement $h(\mathbf{g}(x(n)))$ in a new space composed of vectors with elements consisting of $h(\circ)$ applied to powers of $\mathbf{g}(\mathbf{x}(n))$. The vectors:

$$
\begin{gathered}
\mathbf{y}(n)=\left[h(\mathbf{x}(n)), h\left(\mathbf{g}^{T_{1}}(\mathbf{x}(n))\right), h\left(\mathbf{g}^{T_{2}}(\mathbf{x}(n))\right), \ldots,\right. \\
\left.h\left(\mathbf{g}^{T_{d-1}}(\mathbf{x}(n))\right)\right] .
\end{gathered}
$$

Define a motion in a $d$-dimensional Euclidian space. Several important properties of the observed chaos 
are reproduced in the space $\mathbf{y}(n)$ and the evolution in time $\mathbf{y}(n) \rightarrow \mathbf{y}(n+1)$ follows the unknown dynamics given by Eq. (16). The deterministic behavior of $\mathbf{x}(n) \rightarrow \mathbf{x}(n+1)$ assures the deterministic behavior of the substituting dynamics.

If a general scalar function $h(\circ)$ is considered well as a general function $\mathbf{g}(\mathbf{x})$ consisting of some initial vector and its time delayed versions, then $\mathbf{y}(n)$ is composed simply of time lags of the observation 34 .

$$
\begin{gathered}
\mathbf{y}(n)=[s(n), s(n+T), s(n+2 T), \ldots, \\
s(n+T(d-1))],
\end{gathered}
$$

where $s(n)=h(\mathbf{x}(n))$ and $T_{k}=k T$.

The central issue of this reconstruction is what time lag $T$ and dimension $d$ to use. The time lag may be established by means of the average mutual information (AMI) function working as a nonlinear autocorrelation of the observable. A candidate lag is obtained around the first minimum of this function. Once the lag is determined, a false nearest neighbors (FNNs) Test can be applied over an increasing phase space given by Eq. (18). The dimension that returns the minimum quantity of FNNs is then selected as the embedding dimension of the phase space. For deterministic motion, it is always possible to obtain zero FNN, but if true stochastic data is present in the unknown dynamics, there is some residuary FNN. After the detection of deterministic motion, the estimation of the largest Lyapunov exponent is carried out, which, if positive, confirms the presence of chaos 37

\section{APPLICATION CASE: SAN FRANCISCO}

Crime data analysis supported on the previous framework was performed for the city of San Francisco. A window between 2003 and 2013 was selected for this study. ${ }^{\text {a }}$ Only crime against property identified as burglary was taken into account. These cases were organized chronologically in order to delineate a dynamical perspective. In addition, spatial clustering of cases was considered according to the city organization in districts associated with 10 police stations.

The analysis considers an initial perspective from the stochastic processes theory with the hypothesis of ergodicity. The first characterized process was the daily reported number of cases for the entire city.

$\overline{{ }^{a} \text { Available at https://data.sfgov.org/data?category }}=$ Public\% 20Safety.
The analysis started by looking at the texture of the observable data. Its autocorrelation function was computed in order to estimate the power spectrum density of the process. If long-range dependence and decaying power spectra are detected, then a multifractal analysis is performed to set the necessity of moving toward the reconstruction of the underlying chaotic dynamics supported by Takens theorem.

The Time delta between consecutive criminal events in time was also characterized for each district following the same approach. Time delta is computed as follows:

$$
\mathrm{TD}_{i}=t_{i}-t_{i-1}
$$

where $t_{i}$ is the corresponding time of the $i$ th crime event with $i=1, \ldots, N$. The criminal record time index $i$ goes over the crime events organized chronologically. The sequence $\mathrm{TD}_{i}$ can be understood as an interarrival time process. This kind of process has been studied previously in different application fields, including communication networks, geophysics and biology $[3839$

\subsection{Fractal and Multifractal Analysis Results}

The characterization of daily observed burglary crime data for San Francisco revealed some of the properties described by Ref. 23. In general, results showed mild long-range dependence and a power spectrum that decays as the frequency grows. Although the Hurst exponent of the process confirms a long-range dependence, data ranking of daily reported cases is organized similarly to the step-like Cantor set hierarchy.

Time intervals between consecutive criminal events were also characterized for each district. The power spectra of these processes were compared with harmonic sequence in terms of the mean square error (MSE) in the low frequency range of the spectrum. It was observed that when a spectrum tends to fit the harmonic sequence, its values might be organized according to a $2 n$-rule hierarchy. Regarding both phenomena, a power law with self-similar hierarchy emerged, showing that crime events span similarly over time for all districts.

Initial results concerning the multifractal analysis are summarized from Figs. 1 to 9 . With respect to the daily number of crimes for the entire city (Fig. 1), a mild correlation was observed for the first 100 days; however, the correlation structure debilitated far beyond this window (Fig. 2). In terms 


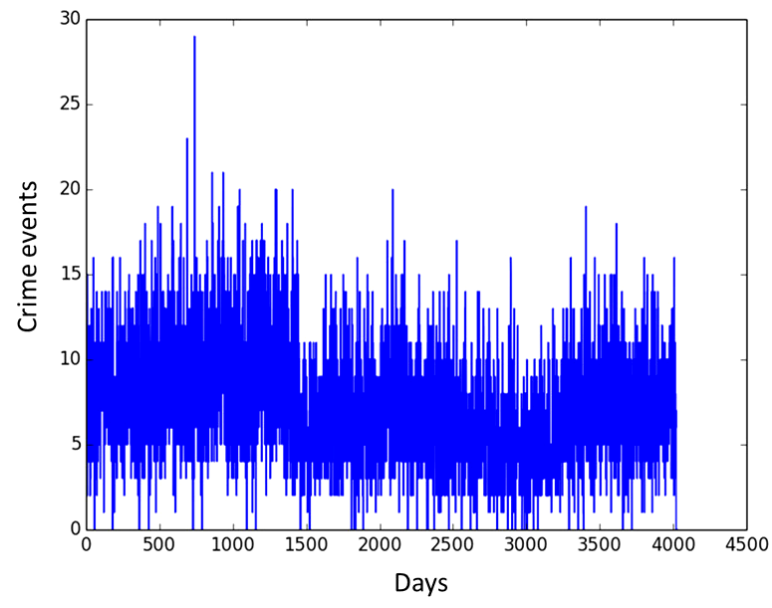

Fig. 1 Daily number of offenses.

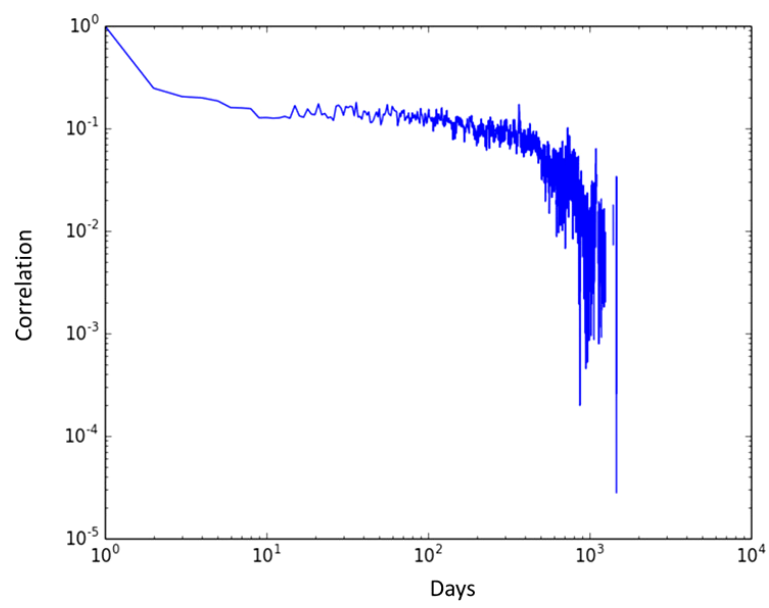

Fig. 2 Autocorrelation function of daily number of offenses.

of the power spectrum analysis, this process exhibited a harmonic structure similar to the $1 / f$ noise (Fig. 3) with an MSE of 0.03 but only for the first decades of the spectrum. The Hurst exponent in this case confirmed the presence of a fractal process with long-range memory (i.e. $H=0.806$ ). However, the disruption suffered by the spectrum in the highest frequencies would suggest the existence of other processes developing simultaneously in other scales. The rank of daily reported crimes (Fig. 4) showed a step-like hierarchy similar to the one of the Cantor set, indicating a fractal organization in the dynamics of the process.

Regarding the characterization of the time delta between consecutive crimes, this is summarized in Table 1 as well as in Fig. 5 for the police districts of San Francisco. The processes with the highest Hurst exponent were likely to cluster in the inner police

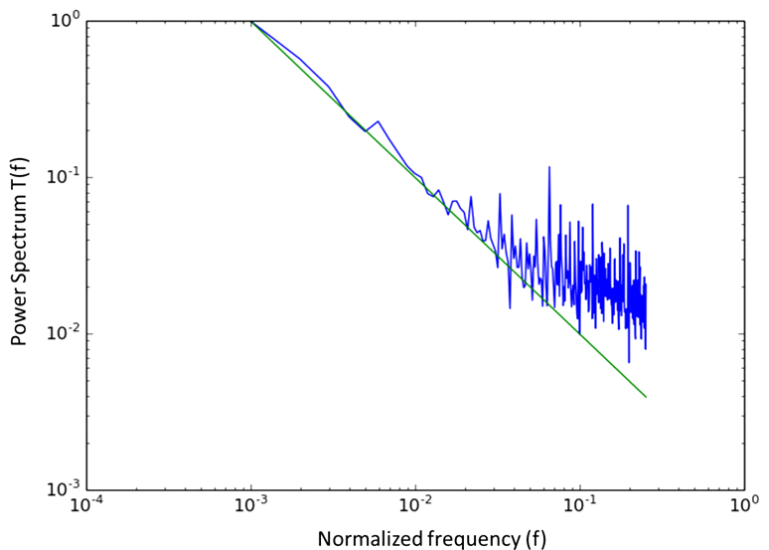

Fig. 3 Power spectra of daily number of offenses.

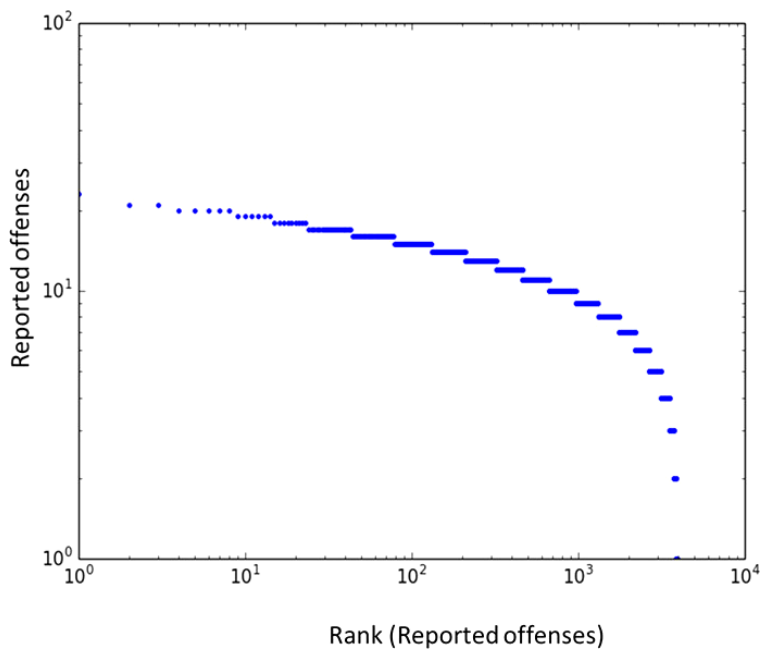

Fig. 4 Rank of daily number of reported offenses.

districts. These districts reported an average number of crimes and some of the smallest MSEs with respect to the $1 / f$ spectrum. The exponent diminished radially from these districts to the periphery of the city, with the exception of one district. In addition, the smallest district also reported the smallest Hurst exponent as well as the smallest number of crimes.

The process with the highest Hurst exponent $(H=0.695)$ is depicted in Fig. 6. This parameter indicated the existence of a long-range memory. It can be also noticed in the correlation structure of the process presented in Fig. 7 A mild correlation was sustained for the first records, which then degenerated. The power density spectrum revealed (Fig. 8) an interesting similarity with the $1 / f$ noise for the first frequency decades $(\mathrm{MSE}=0.013)$. Finally, the rank of process values 
Table 1 Results of Fractal Analysis for Time Delta Between Consecutive Offenses.

\begin{tabular}{lcccc}
\hline $\begin{array}{l}\text { Police } \\
\text { District }\end{array}$ & $\begin{array}{c}\text { Number of } \\
\text { Cases }\end{array}$ & $\begin{array}{c}\text { Hurst } \\
\text { Exponent }(\boldsymbol{H})\end{array}$ & $\begin{array}{c}\text { Information } \\
\text { Dimension } \boldsymbol{D}(\mathbf{1})\end{array}$ & $\begin{array}{c}\text { MSE } \\
(\mathbf{1} / \boldsymbol{f} \text { Noise })\end{array}$ \\
\hline MISSION & 6,390 & 0.6842 & 0.9952 & 0.017 \\
NORTHERN & 10,284 & 0.6455 & 0.9955 & 0.043 \\
CENTRAL & 7,764 & 0.6503 & 0.9948 & 0.015 \\
PARK & 5,206 & 0.6955 & 0.9945 & 0.013 \\
RICHMOND & 4,753 & 0.6497 & 0.9999 & 0.011 \\
BAYVIEW & 7,191 & 0.6144 & 0.9978 & 0.055 \\
SOUTHERN & 8,442 & 0.5952 & 0.9913 & 0.128 \\
TARAVAL & 6,174 & 0.6013 & 0.9959 & 0.033 \\
INGLESIDE & 5,809 & 0.5872 & 0.9920 & 0.101 \\
TENDERLOIN & 2,697 & 0.5031 & 0.9981 & 0.305 \\
\hline
\end{tabular}

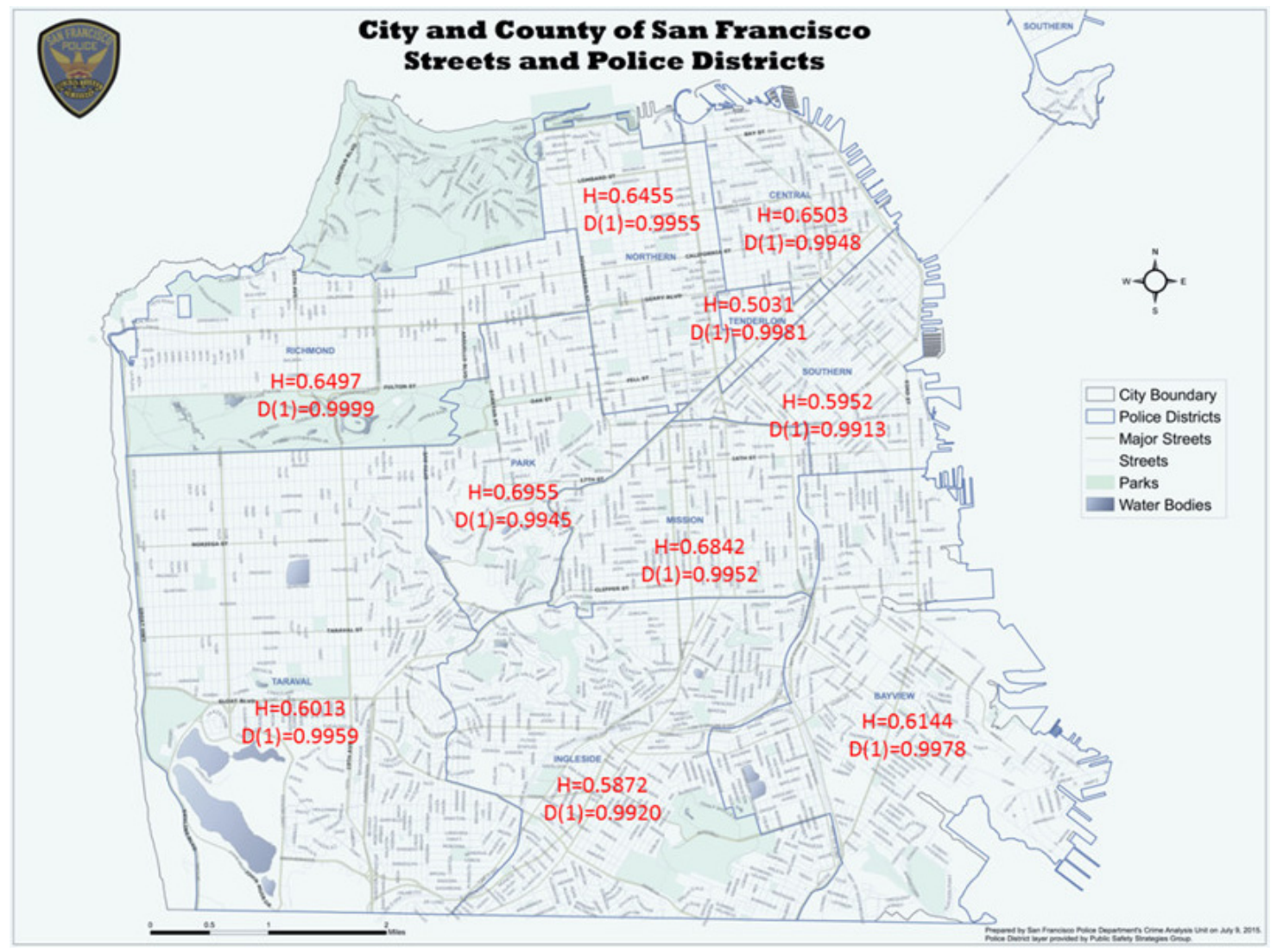

Fig. 5 Hurst exponent and information dimension of the time delta between consecutive crimes over the police districts. Source: The San Francisco police districts map is provided on-line by the San Francisco police department at http://sanfranciscopolice.org/police-district-maps.

shaped a distribution with no steps; however, it was not possible to fit it to a zipf's law since the resulting exponent was smaller than one (Fig. 9).

Figure 10 presents a hierarchical power law that relates the number of crimes per district and the average time between consecutive crimes per district. The existence of such a power law is also supported in Table 2 Each district is assigned to a class in the district hierarchy, which is constructed by organizing the districts according to their quantity of reported offenses from the smallest (class 1) to the highest (class 10). The average time between consecutive crimes multiplied by the total number of crimes per district tends to be a constant for all districts similar to the total time span registered from 2003 to 2013. In addition, the ratio RD tends 


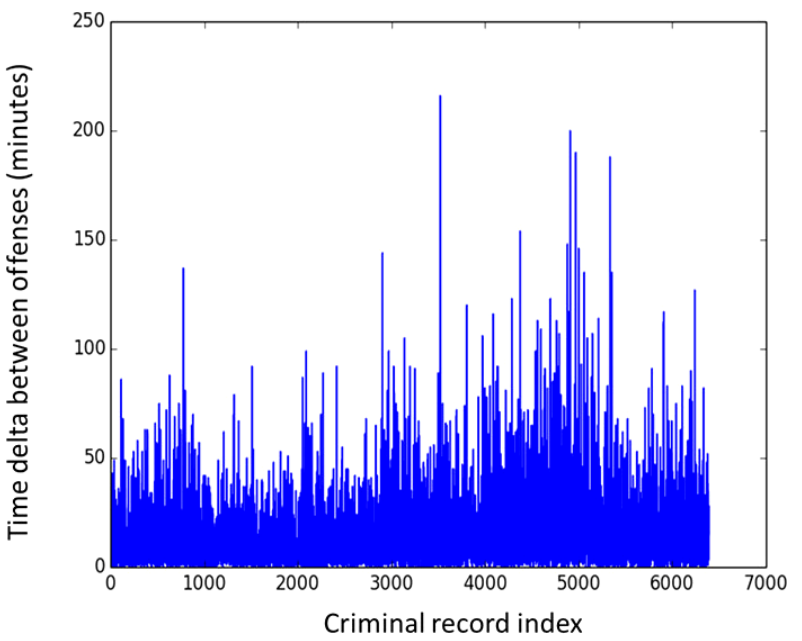

Fig. 6 Time delta between consecutive offenses (district with the highest Hurst exponent).

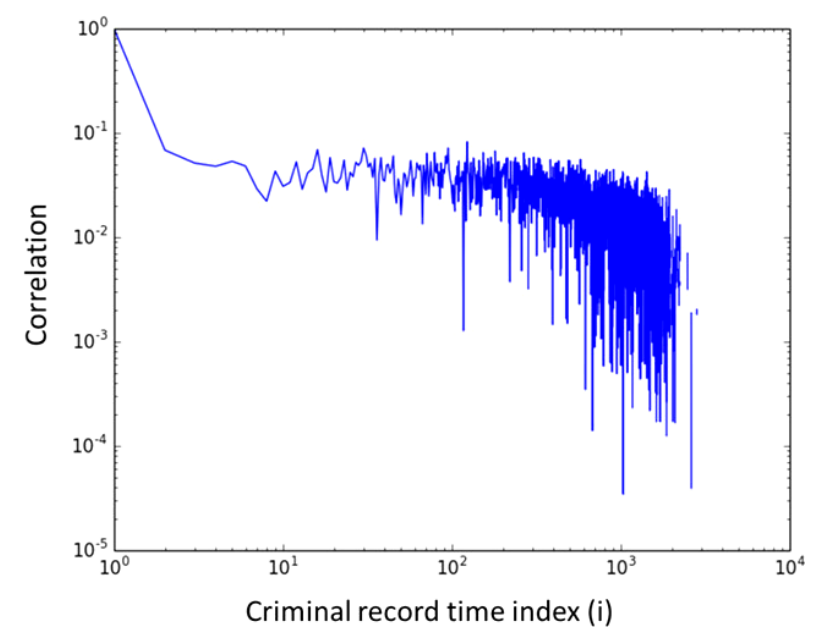

Fig. 7 Autocorrelation function of time delta between consecutive offenses.

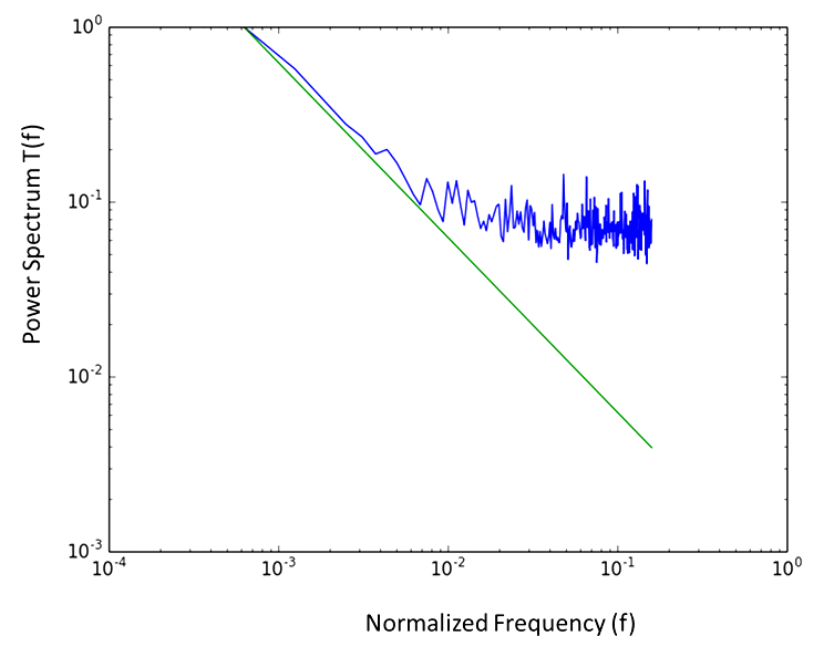

Fig. 8 Power spectra time delta between consecutive offenses.

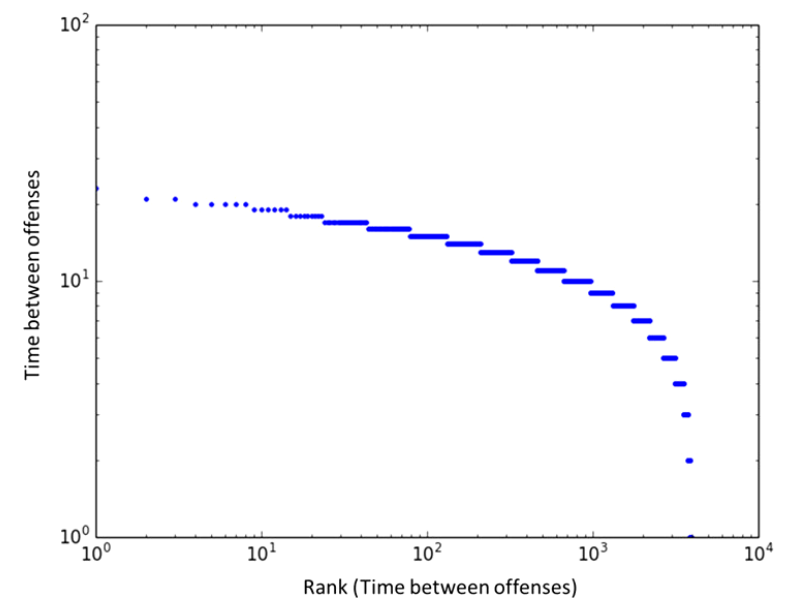

Fig. 9 Rank time delta between consecutive offenses.

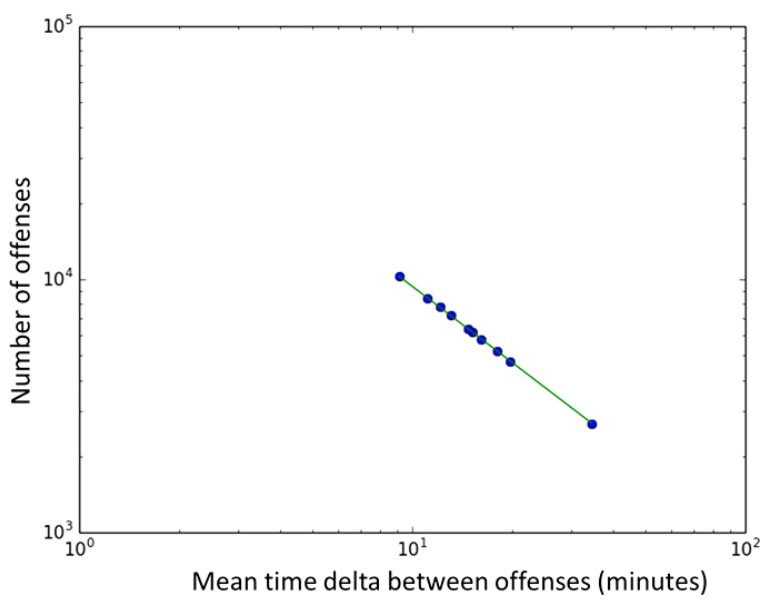

Fig. 10 Hierarchical power law emerging from burglary records in San Francisco that suggests multifractal behavior.

to one, with

$$
\mathrm{RD}=\ln (\mathrm{Rn}) / \ln (\mathrm{Ra}),
$$

where $\mathrm{Rn}$ is the ratio between the number of crimes per class and $\mathrm{Ra}$ is the ratio between times per class. The exponent of the power law is also close to one with

$$
C(t)=4.976 t^{-1.0035} \text {. }
$$

Thus, the existence of a rank-size rule was confirmed, and it also behaved like a self-similar hierarchy.

Previous results show that crime offenses in San Francisco exhibit multifractal organization in time. This observation is also validated in Fig. 11, which depicts the multifractal spectrum obtained for the processes with the highest and lowest Hurst exponents. The spectra were computed by using a discrete wavelet decomposition of this signal 40 
Table 2 Description of the Results Observed in the Hierarchical Power Law That Relates the Quantity of Crimes in a District to the Average Time Between Consecutive Crimes.

\begin{tabular}{rrrccc}
\hline Classes & Offenses & $\begin{array}{c}\text { Sum of Time } \\
\text { Deltas }\end{array}$ & $\begin{array}{c}\text { Average Time } \\
\text { Delta }\end{array}$ & $\begin{array}{c}\text { Amount Ratio Between } \\
\text { Classes (Ra) }\end{array}$ & $\begin{array}{c}\text { Time Ratio Between } \\
\text { Classes (Rn) }\end{array}$ \\
\hline 1 & 2,697 & 93,472 & 34.658 & & 1.759 \\
2 & 4,753 & 93,602 & 19.693 & 1.762 & 1.093 \\
3 & 5,206 & 93,715 & 18.001 & 1.095 & 1.185 \\
4 & 5,809 & 93,656 & 16.122 & 1.186 & 1.061 \\
5 & 6,174 & 93,745 & 15.183 & 1.062 & 1.0351 \\
6 & 6,390 & 93,731 & 14.668 & 1.035 & 1.125 \\
7 & 7,191 & 93,686 & 13.028 & 1.125 & 1.077 \\
8 & 7,764 & 93,885 & 12.092 & 1.080 & 1.087 \\
9 & 8,442 & 93,905 & 11.123 & 1.087 & 1.218 \\
10 & 10,284 & 93,860 & 9.126 & 1.218 & \\
\hline
\end{tabular}

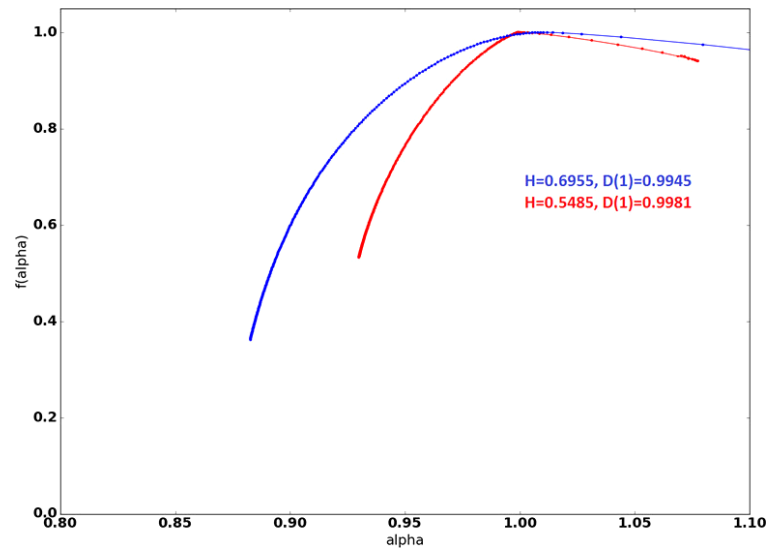

Fig. 11 Multifractal spectra for time delta between consecutive offenses (districts with the highest and lowest Hurst exponent in blue and red, respectively).

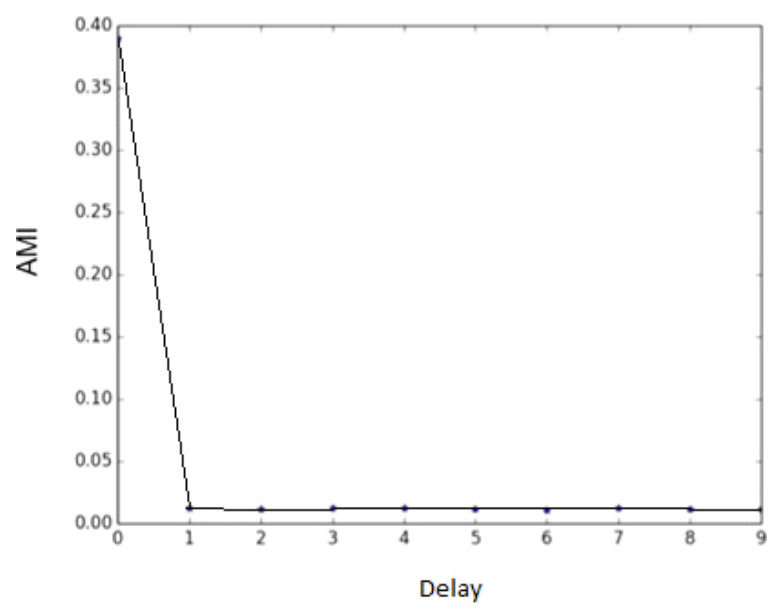

Fig. 12 AMI for time delta between consecutive offenses.

These span over a wide range of local dimensions, revealing the presence of multiple processes running in different time scales. The information dimension $D(1)$ in both cases is very close to one, leading one to inquire into the possible deterministic chaotic behavior of the interarrival time processes. This dimension is also highlighted in Fig. 5 for all processes, which behave in a similar way with respect to this statistical attribute.

\subsection{Results from Observed Chaos}

The AMI of the crime signal shown in Fig. 6]reports a first minimum in $T=2$, as can be seen in Fig. 12 . This result indicates that the phase space reconstruction is possible with this delay according to Eq. (18). The next statistical attribute was an FNN test whose results are plotted in Fig. 13. FNN goes to zero for $d=4$, showing that this crime signal has a deterministic nature associated with a $4 \mathrm{D}$ dynamical system. Once the delay and dimension were known, the maximum Lyapunov exponent

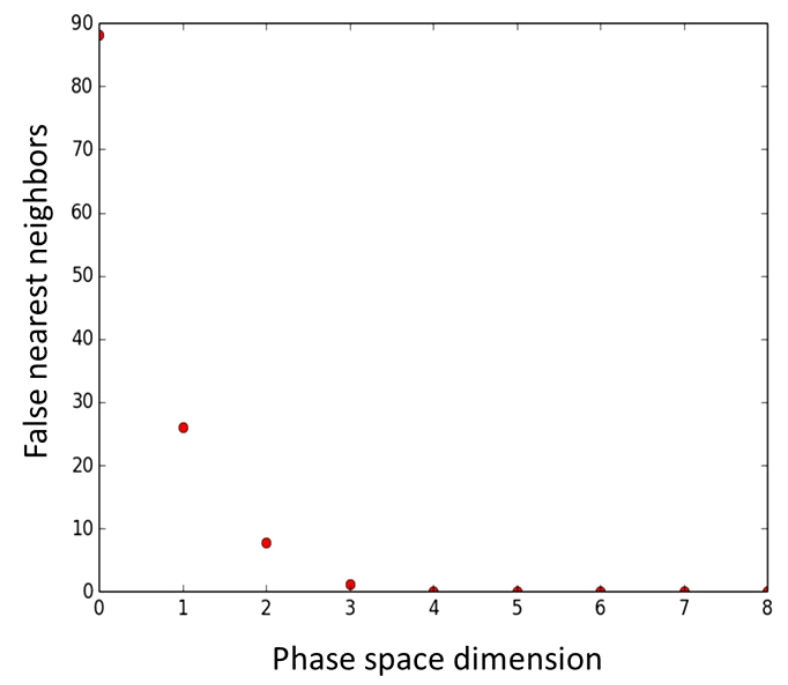

Fig. 13 FNNs for time delta between consecutive offenses revealing a fourth dimensional phase space. 
was estimated as shown in Fig. 14. The obtained estimation shows an increasing behavior, which can be fitted as a linear regression with positive slope. This result confirms that chaos is present in

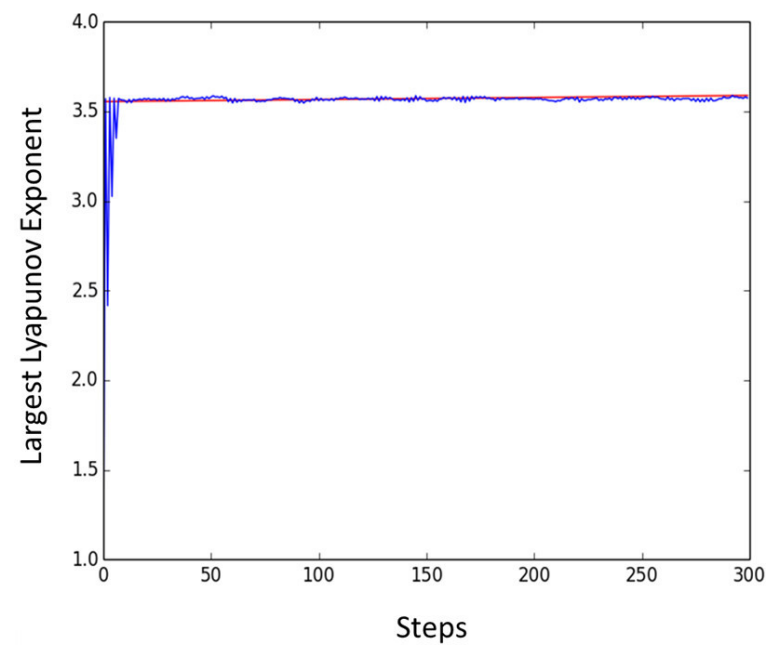

Fig. 14 Largest Lyapunov exponent estimation for time delta between consecutive offenses confirming the presence of chaotic dynamics.

\begin{tabular}{|c|c|c|c|c|c|c|c|c|c|c|}
\hline$\langle(t-l)$ & $x(t-3 l)$ & $X(t)$ & $x(t-l)$ & $X(t-2 l)$ & $x(t-3)$ & $X(t)$ & $\mathrm{X}(\mathrm{t}-21)$ & $x(t-l)$ & $\mathrm{X}(t)$ & $x(t-3 l)$ \\
\hline 1 & 0 & 1 & 1 & 0 & 0 & 1 & 0 & 1 & 1 & 0 \\
\hline 0 & 0 & 1 & 0 & 1 & 0 & 1 & 1 & 0 & 1 & 0 \\
\hline
\end{tabular}

(a)

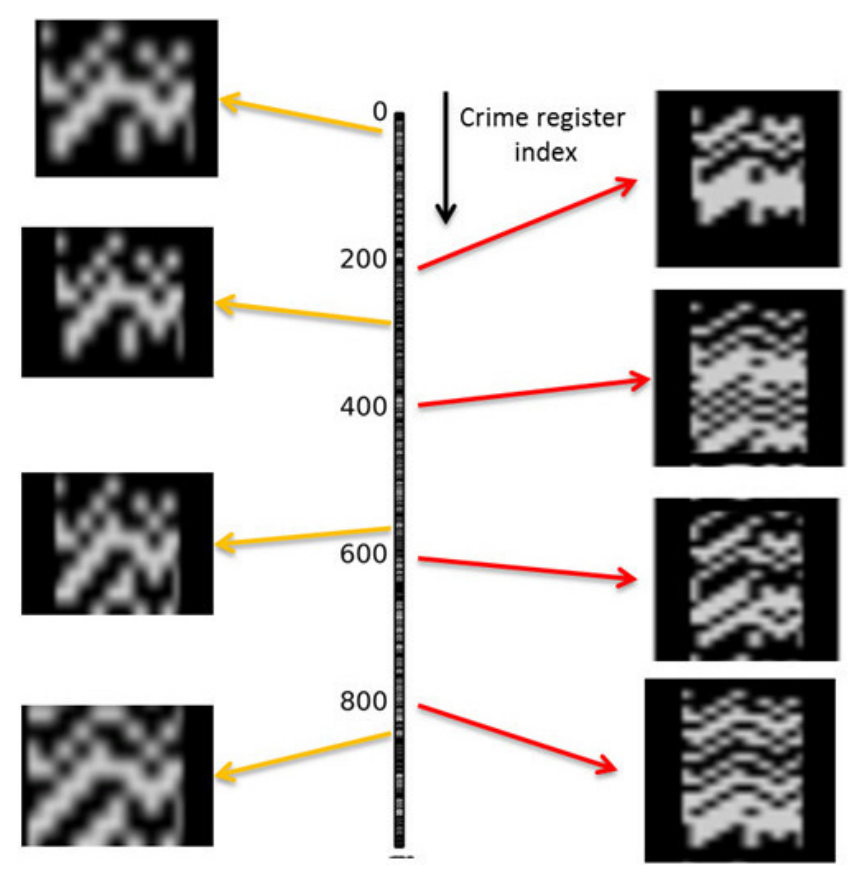

(b)

Fig. 15 (a) Informational phase space organization, (b) informational patterns that emerge from the chaotic dynamics of time delta between consecutive offenses. the dynamics of the time delta between criminal events.

The visualization of a $4 \mathrm{D}$ phase space can be quite complex. Taking into account that a chaotic system can be understood as an information source producing symbols, $\frac{19}{19}$ we decided to represent an informational phase space, obtained from a coarse grained representation of the time delta between criminal events. This representation is depicted in Fig. [15]. Each variable of the phase space is compared with its mean valuable for each crime record register. If the time delta of a given criminal record is greater than the mean, a symbol "1" is produced; if not, a symbol "0" is obtained. A sample of this process is depicted in Fig. 15b for the first thousand criminal registers of Fig. 6. It can be observed that two interesting patterns of symbols (i.e. system words) appear several times, which indicates that at least in this window - recurrence is observed in the chaotic dynamics of the observable.

\section{DISCUSSION AND CONCLUSION}

\subsection{General Considerations}

A quantification of the complex dynamics of criminal activity has been considered in this work. This attempt is intended to propose an analysis framework in criminology that takes advantage of fractal and multifractal analysis as well as chaos and information theory. Stochastic process theory is a valuable tool to approach the analysis of criminal patterns; however, it should be considered only as a first step in a deeper explicative analysis.

The existence of multifractality in criminal phenomena, as considered in this work, leads to inquiry beyond the stochastic point of view. The next step in this inquiry would be to move the question of criminal patterning to the dynamical systems theory, particularly dealing with the problem of chaos quantification, modeling and possible prediction in criminal data. Another possibility would be to understand criminal activity as information sequences (i.e. words) produced by complex systems. The problem of anticipating criminal patterns would correspond to the one of predicting such words from a coded information source.

Regarding the city of San Francisco, criminal records in terms of interarrival processes reflected the footprint of complexity as the presence of fractal and multifractal behavior. Several observations 
support this claim. First, the computed Hurst exponent for all police districts was greater than 0.5. Second, a hierarchical power law that correlates the number of crimes and the mean time between crime events emerged from this administrative division. This evidence suggests that in terms of stochastic processes, the nature of criminal events should be revisited. The question about the underlying processes behind criminal activity is still open in this sense. More research is needed around the distribution of crime in time; in particular, the notion of Poisson-distributed events 141 may be examined, introducing other kind of models such as multiplicative cascades. $\underline{39}$

Evidence that interarrival processes of criminal activity in San Francisco behave similarly to $1 / f$ noise was also presented in terms of the observed spectra, long-range dependences and step-like rankings. This kind of analysis may be performed for other cities in order to look at the generality of these observations. If more supporting evidence is accumulated, this would lead to inquiry about the nature of crime itself as a complex phenomenon. Thus, SOC and HOT theories might be interrelated to environmental criminology as a complementary way of explaining the complexities of crime dynamics.

Regarding the characterization of the chaotic behavior of the interarrival time processes of criminal activity, a high-dimensional phase space was detected for the district with the largest Hurst exponent. This evidence opens the door to exploring predictive models operating over the phase space of the dynamics and the use of high-dimensional data techniques to understand the temporal patterns of crime dynamics.

\subsection{Chaos, Rules and Crime}

The informational patterns presented in Fig. 15b reveal several interesting artifacts that appear frequently over the attractor dynamics shaped by the phase space. Some artifacts are depicted in Fig. 16, showing the inherently chaotic or complex (i.e. edge of chaos) behavior of the dynamics. These artifacts have been identified and studied in 1D and 2D cellular automata simulations, among these are found: complex structures, spaceships, gliders and diagonal lines 42 Although these artifacts emerge from automata, the rules behind them are well established. In the case of spaceships and gliders, 2D life-like cellular automata can produce this kind of artifacts, while diagonal lines can be produced by simple 1D automata. Wolfram evidenced that 1D cellular automata can be classified in four universality classes, particularly class 3 exhibits aperiodic chaotic patterns, while class 4 is capable of propa-

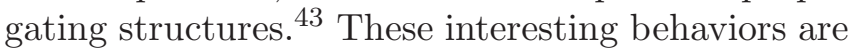
obtained from simple rules and initial conditions in both cases.

The rules behind artifacts in cellular automata are expressed in simple ways. For example, rule 30 (Fig. 17a) in 1D Wolfram's automata is able to produce diagonal lines, as shown in Fig. 17b. It has been connected to the production of fractal patterns over long range simulations (Fig. 17k). Other $1 \mathrm{D}$ rules like 184 and 90 can also produce vertical lines as well as interesting patterns. The rule B3S23 (i.e. Born with three neighbors and Survive with two or three neighbors) used in Conway's game of life produces spaceships and gliders among other artifacts. While large complex structures are found among the artifacts produced by rules like B1S1, which is classified as a larger than life rule.44
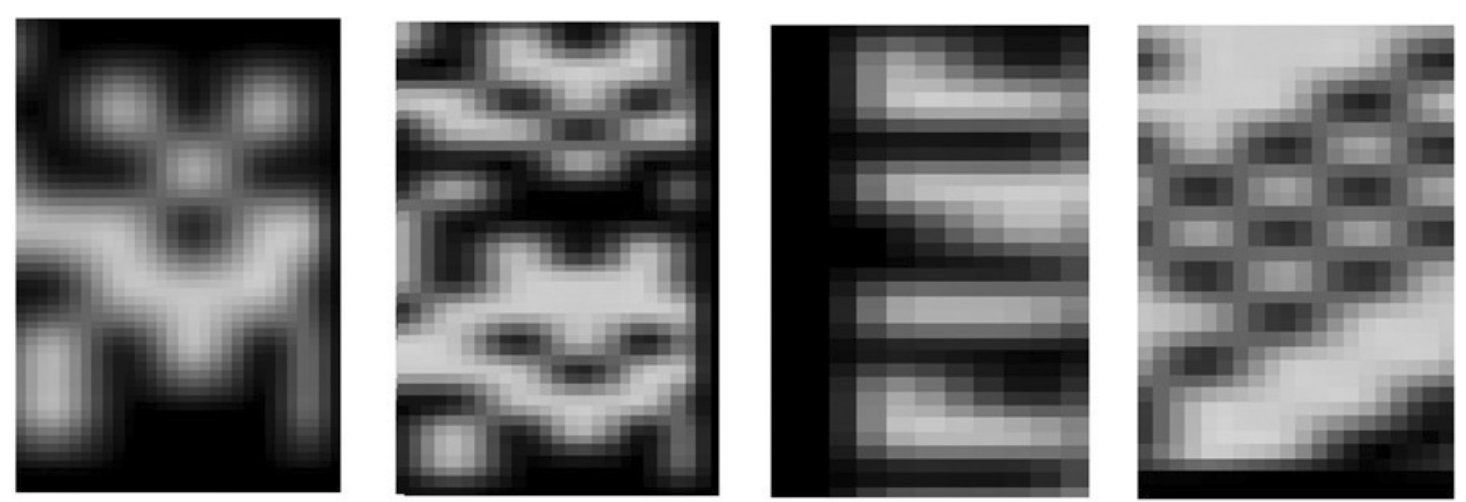

Fig. 16 Detected artifacts, from left to right: large complex structures, spaceships, gliders and diagonal lines. 


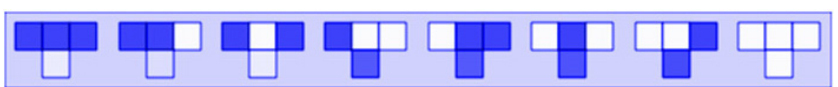

(a)

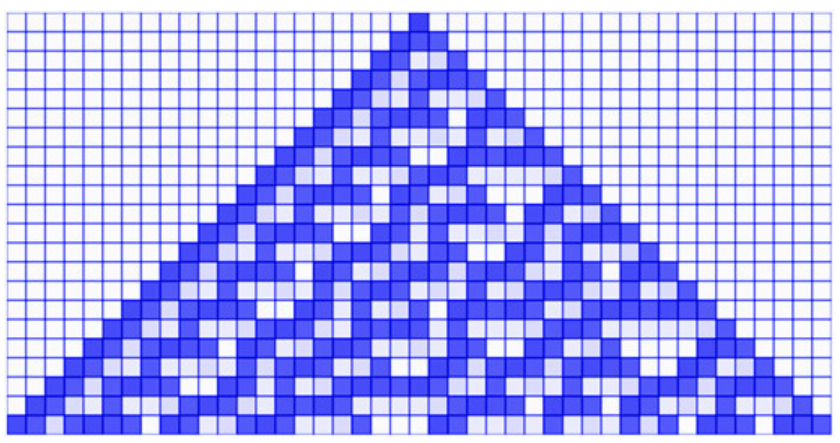

(b)

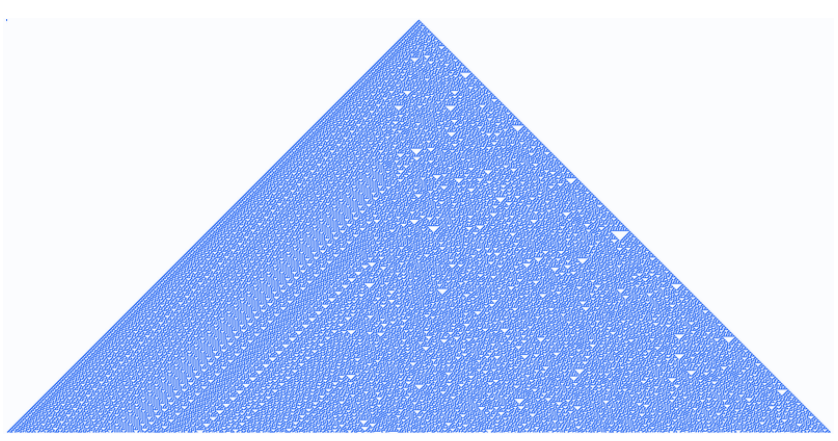

(c)

Fig. 17 (a) Rule 30, (b) 1D cellular automaton computing rule 30 during some steps, (c) long range simulation of the 1D cellular automaton with rule 30 . Taken from Wikipedia under Creative Commons license.

These rules are in general simple and produce complex artifacts. The associated patterns are difficult to predict and in some cases emerge from long simulations. Besides, the logic behind these rules seems to be in concordance with minimum energy principles or predator-prey games. $\stackrel{45}{-}$ In this sense, we suggest here that some of these rules may be considered as potential models of crime dynamics at least in informational terms.

As the informational patterns appear through a coarse grained representation of the interrarrival time process, a bit of information is produced if the time delta between consecutive crimes tends to be small or large. On the other hand, the combination of small and large time deltas may or not produce information. The arrival of a new crime that produces information is expected if crimes have occurred in recent or distant past. Rule 30 in 1D wolfram automaton considers a similar behavior, as shown in Fig. 17 , note that a white dot is produced either three white or black dots appear.

Cellular automata for simulating the time dynamics of criminal activity is suggested as a way to discover underlying rules. These rules should not be understood as stationary, rigid and definitive constructions. Social systems are characterized by its capability of producing new or combining existing rules $\frac{46}{6}$ Thus the search for simple rules behind crime dynamics supported on computational and logical principles would require the development of complete epistemological and methodological framework in which rule innovation is possible. In this framework, detecting chaos in crime dynamics is a critical task. A selected set of nonlinear statistics, as the one considered in this work, should be in the core of the required detection.

\subsection{Implications on Policy, Prevention and Control}

Public policing over social systems, related to the production of crime dynamics as the one studied in this work, should recognize their nature. These systems evolve continuously by generating new knowledge in terms of rule innovations that survive over time. ${ }^{47}$ This consideration in addition to the results presented in this work suggests that dynamic policies should be formulated in order to perform some sort of prevention and control. These policies can be formulated over a time span considering what is the most probable rule or combination of rules.

Possible patterns as informational artifacts that emerge from simulation scenarios may lead the formulation of required strategies. If a rule is mined from recent criminal records, several simulations considering different initial conditions can be computed in order to depict possible scenarios of crime occurrence. If the artifacts reveal substantial production of information as in the case of gliders or long diagonal lines, crime alert networks may broadcast preventive bulletins more frequently or additional patrol agents can be deployed.

The idea of crime anticipation based on informational artifacts detection is to correlate the amount of information produced by a crime system with the corrective actions to perform control over it. Information production does not anticipate the exact occurrence of crimes, but suggests some approximation to the rate at which they appear. Therefore, feedback can be achieved since time delays are reduced due to the introduced anticipation and 
plausible actions can be produced by some control law. Anticipatory schemes for crime prevention are recently developed in literature operating from different sources of information,,$\frac{48 \mid 49}{b}$ being a promising research area.

\section{REFERENCES}

1. M. R. D'Orsogna and M. Perc, Statistical physics of crime: A review, Phys. Life Rev. 12 (2014) 1-21.

2. P. J. Brantingham and P. L. Brantingham, Crime pattern theory, in Environmental Criminology and Crime Analysis (Willian Publishing, 2008), pp. 7893.

3. P. L. Brantingham, U. Glasser, P. Jackson, B. Kinney and M. Vajihollahi, Mastermind: A computational modeling and simulation of spatiotemporal aspects of crime in urban environments, in Artificial Crime Analysis Systems: Using Computer Simulations and Geographic Information Systems (Information Science Reference, 2008), pp. 252-280.

4. M. Felson, Routine activity approach, in Environmental Criminology and Crime Analysis (Taylor \& Francis, 2008), pp. 70-77.

5. J. H. Ratcliffe, A temporal constraint theory to explain opportunity-based spatial offending patterns, J. Res. Crime Delinq. 43(3) (2006) 261-291.

6. V. Furtado, A. Melo, A. L. V Coelho, R. Menezes and R. Perrone, A bio-inspired crime simulation model, Decis. Support Syst. 48(1) (2009) 282-292.

7. G. Mohler and M. Short, Geographic profiling from kinetic models of criminal behavior, Siam J. Appl. Math. 72(1) (2012) 163-180.

8. S. J. Rey, E. A. Mack and J. Koschinsky, Exploratory space-time analysis of burglary patterns, J. Quant. Criminol. 28(3) (2012) 509-531.

9. G. Mohler, Marked point process hotspot maps for homicide and gun crime prediction in Chicago, Int. J. Forecast. 30(3) (2014) 491-497.

10. T. H. Grubesic, On the application of fuzzy clustering for crime hot spot detection, J. Quant. Criminol. 22(1) (2006) 77-105.

11. P. Brantingham, P. Brantingham, M. Vajihollahi and K. Wuschke, Crime analysis at multiple scales of aggregation: A topological approach, in Putting Crime in its Place (Springer, NY, 2009), pp. 87-107.

12. T. Davies and S. D. Johnson, Examining the relationship between road structure and burglary risk via quantitative network analysis, J. Quant. Criminol. 31(3) (2015) 481-507.

13. G. D. Walters, Crime and chaos: Applying nonlinear dynamic principles to problems in criminology, Int. J. Offender Ther. Comp. Criminol. 43(2) (1999) 134-153.
14. M. Short, A. Bertozzi and P. Brantingham, Nonlinear patterns in urban crime: Hotspots, bifurcations and suppression, SIAM J. Appl. Dyn. Syst. 9(2) (2010) 462-483.

15. N. Rodríguez, On the global well-posedness theory for a class of PDE models for criminal activity, Physica D 260 (2013) 191-200.

16. R. Frank, V. Dabbaghian, A. Reid, S. Singh, J. Cinnamon and P. Brantingham, Power of criminal attractors: Modeling the pull of activity nodes, J. Artif. Soc. Soc. Simul. 14(6) (2011) 1-27.

17. N. Malleson and M. A Andresen, Spatio-temporal crime hotspots and the ambient population, Crime Sci. 4(1) (2015) 10.

18. J. H. Holland, Complex adaptive systems, Daedalus 121(1) (1992) 17-30.

19. G. Nicolis and C. Nicolis, Foundations of complex systems, European Rev. 17(2) (2009) 237-248.

20. Y. Bar-Yam, Dynamics of Complex Systems (Addison-Wesley, Reading, MA, 1997).

21. M. Mitchell, Complexity: A Guided Tour (Oxford University Press, 2009).

22. P. Sice and I. French, A holistic frame-of-reference for modelling social systems, Kybernetes $\mathbf{3 5}(6)$ (2006) 851-864.

23. Y. Chen, Zipf's law, $1 / f$ noise and fractal hierarchy, Chaos Solitons Fractals 45(1) (2012) 63-73.

24. L. M. A. Bettencourt, J. Lobo, D. Strumsky and G. B. West, Urban scaling and its deviations: Revealing the structure of wealth, innovation and crime across cities, PLoS One 5(11) (2010) 20-22.

25. M. Newman, Power laws, Pareto distributions and Zipf's law, Contemp. Phys. 46(5) (2005) 323351.

26. P. Bak, C. Tang and K. Wiesenfeld, Self-organized criticality: An explanation of the $1 / f$ noise, Phys Rev. Lett. 59(4) (1987) 381-384.

27. J. Feder, Fractals (Plenum Press, New York, 1998).

28. C. Puentes and N. Obregon, A deterministic geometric representation of temporal rainfall: Results for a storm in Boston, Water Resour. Res. 32(9) (1996) 2825-2839.

29. N. Obregón, C. E. Puente and B. Sivakumar, Modeling high-resolution rain rates via a deterministic fractal-multifractal approach, Fractals 10(3) (2002) 387-394.

30. J. M. Carlson and J. Doyle, Highly optimized tolerance: A mechanism for power laws in designed systems, Phys. Rev. E 60(2) (1999) 1412-1427.

31. D. Ricker, Echo Signal Processing (Kluwer Academic Publishers, 2003).

32. T. Halsey, M. Jensen, L. Kadanoff, I. Procaccia and B. Shraiman, Fractal measures and their singularities, Phys. Rev. A 33(2) (1986) 1141-1151.

33. J. De Souza and S. Pires Rostirolla, A fast MATLAB program to estimate the multifractal spectrum 
of multidimensional data: Application to fractures, Comput. Geosci. 37(2) (2011) 241-249.

34. H. Abarbanel, Analysis of Observed Chaotic Data (Springer-Verlag, New York, 1996).

35. H. D. I. Abarbanel, R. Brown, J. J. Sidorowich and L. S. Tsimring, The analysis of observed chaotic data in physical systems, Rev. Mod. Phys. 65(4) (1993) 1331-1392.

36. F. Takens, Detecting strange attractors in turbulence, Dyn. Syst. Turbul. Lect. Notes Math. 898 (1981) 366-381.

37. M. T. Rosenstein, J. J. Collins and C. J. De Luca, A practical method for calculating largest Lyapunov exponents from small data sets, Physica D 65(1-2) (1993) 117-134.

38. G. Wornell, Wavelet-based representation for the 1/f family of fractal processes, Proc. IEEE 81(10) (1993) 1428-1450.

39. R. H. Riedi, M. S. Crouse, V. J. Ribeiro and R. G. Baraniuk, A multifractal wavelet model with application to network traffic, IEEE Trans. Inf. Theory 45(3) (1999) 992-1018.

40. R. Lopes and N. Betrouni, Fractal and multifractal analysis: A review, Med. Image Anal. 13(4) (2009) 634-649.

41. M. B. Short et al., A statistical model of criminal behavior, Math. Model. Methods Appl. Sci. 18(Suppl.) (2008) 1249-1267.
42. S. Wolfram, A New Kind of Science (Wolfram Media, 2002).

43. S. Wolfram, Universality and complexity in cellular automata, Physica D 10 (1984) 1-35.

44. K. M. Evans, Larger than life: Digital creatures in a family of two-dimensional cellular automata, Discrete Mathematics and Theoretical Computer Science Proc. (2001), pp. 177-192.

45. G. Cattaneo, A. Dennunzio and F. Farina, A full cellular automaton to simulate predator-prey systems, in 7th Int. Conf. Cellular Automata (2006), pp. $446-451$.

46. K. Dopfer, The economic agent as rule maker and rule user: Homo Sapiens Oeconomicus, J. Evol. Econ. 14(2) (2004) 177-195.

47. C. Olaya, L. Guzmán and J. Gomez-quintero, An engineering perspective for policy design: Selforganizing crime as an evolutionary social system, Trends Organ. Crime (2016).

48. A. Bogomolov, B. Lepri, J. Staiano, N. Oliver, F. Pianesi and A. (Sandy) Pentland, Once upon a crime: Towards crime prediction from demographics and mobile data, in Icmi $^{\prime} 14$ (2014), pp. 427-434.

49. M. S. Gerber, Predicting crime using Twitter and kernel density estimation, Decis. Support Syst. 61 (2014) 115-125. 